\title{
New Approach to Ultracapacitor Technology: What it Can Offer to Electrified Vehicles
}

\author{
Yurii Maletin $^{1,2}$, Natalia Stryzhakova ${ }^{1,2}$, Sergii Zelinskyi ${ }^{1,2}$, Sergey Chernukhin ${ }^{1}$, Dmytro Tretyakov ${ }^{1,2}$, Hugo \\ Mosqueda $^{1}$, Natalia Davydenko ${ }^{1}$ and Dmytro Drobnyi ${ }^{1,2}$ \\ 1. YUNASKO-UKRAINE Limited Liability Company, Kiev 03164, Ukraine \\ 2. Institute for Sorption and Problems of Endoecology, Ukraine Academy of Sciences, Kiev 03164, Ukraine
}

Received: March 09, 2015 / Accepted: April 14, 2015 / Published: June 30, 2015.

\begin{abstract}
Novel carbon-carbon ultracapacitors and hybrid lithium-carbon devices are described. New approach to the design of electrode materials and electrochemical systems followed by the improved design of ultracapacitor cells and modules have resulted in prototypes of superior performance that was verified by independent tests in the Institute of Transportation Studies, UC (ultracapacitor) Davis, in JME Inc., in Wayne State University, and in some other labs. All the test results confirm the superlative performance of the devices developed: carbon-carbon ultracapacitors demonstrate the extremely low inner resistance resulting in the highest power capability and efficiency that also alleviates the cooling requirements and improves safety. Our "parallel" hybrid devices demonstrate substantially higher energy density than competing LIC (lithium ion capacitor) technologies keeping at the same time the high power density, comparable with the best carbon-carbon ultracapacitors available in the market. In order to make ultracapacitor technology even more attractive to automakers, new organic electrolytes (not ionic liquids) have been developed and are currently under testing at temperatures about $100^{\circ} \mathrm{C}$ and voltages up to $3.0 \mathrm{~V}$.
\end{abstract}

Key words: Ultracapacitor, hybrid device, high power, large energy.

\section{Introduction}

The electrochemical double layer capacitor or UC (ultracapacitor) market is rapidly gaining momentum over the past decade, in particular, in automotive sector. An increased market of electric and hybrid buses (in particular, in China) is supported by the successful use of UC's in stop \& start systems of Peugeot e-HDI, in regenerative braking system of Mazda i-ELOOP, in Toyota safety braking system and some other applications. On the other hand, the automotive industry puts forward very stringent requirements as to the performance of UC devices. As an unambiguous UC advantage, their long cycle life is worth noting as this can be of importance for some automotive applications related with frequent

Corresponding author: Yurii Maletin, Ph.D., Dr. habil., research fields: physical chemistry and energy storage. E-mail: ymaletin@yunasko.com. charge-discharge events, e.g., in stop \& start or KERS (kinetic energy recovery systems). An obvious disadvantage of UC devices is their low energy density as compared with batteries - typically of the order of 4-5 Wh/kg. Therefore, UCs are normally considered not as energy sources but rather as power supply units capable of delivering high bursts of power on discharge and capable of being charged very fast (within seconds). This is due to their extremely low inner resistance, if compared with batteries, and the low resistance also results in high efficiency and low heat generation, hence, in improved safety. Nevertheless, further reduction in UC inner resistance and, correspondingly, further increase in UC efficiency is desirable, in particular, in combined systems, wherein, UC is connected parallel to battery. In this case, the lower is the UC resistance the more effectively the battery can be unloaded as shown below. 
On the other hand, an increase in UC energy density would also be very much desirable and this can be achieved with the use of hybrid technologies if the electrochemical system contains the components of both UC and battery technology.

Though UC devices, in particular, those based on acetonitrile electrolytes can effectively be used at low temperatures, their upper temperature limit of $65-70{ }^{\circ} \mathrm{C}$ is considered as a drawback and should be increased up to $90-100{ }^{\circ} \mathrm{C}$ to allow them to be placed not far from the car engine or to be used in hot countries. As still another challenge, an increase in rated voltage from typical today's value of 2.5-2.7 V up to $3+\mathrm{V}$ should be taken into account.

All the key points mentioned above are thoroughly studied in our lab today and some results are briefly discussed in this paper.

\section{Experimental}

\subsection{Design of Prototypes}

Two-electrode prototypes were used to measure the performance of various components and design solutions. The electrodes were typically prepared by mixing the active material and conductive additive powder with PTFE (polytetrafluoroethylene) suspension in water (the PTFE was used as a binder) until a homogeneous mixture was obtained. Commercially available activated carbons, mostly those produced by Kuraray in Japan (YP50F or YP80F) or by Haycarb in Sri Lanka (HDLC 20B STUW), were used for UC or hybrid prototype manufacture. Some experimental samples of activated carbons developed in our lab were also tested for comparison purposes. Additionally, in hybrid devices, LTO $\left(\mathrm{Li}_{4} \mathrm{Ti}_{5} \mathrm{O}_{12}\right)$ was used as an active material for negative electrodes, and a mixture of $\mathrm{Li}_{x} \mathrm{Mn}_{2} \mathrm{O}_{4}$ and $\mathrm{LiFePO}_{4}$ was used to manufacture positive electrodes. A mixture of active materials and the binder was rolled to form sheets of 50-100 micron thick followed by cutting off the separate electrodes. The electrodes thus obtained had their geometric surface area of $15 \mathrm{~cm}^{2}$ or $35 \mathrm{~cm}^{2}$ each. They were then applied onto electric-spark treated $[1,2]$ aluminum foil (current collector) of 15 or 20 micron thick and dried at $220^{\circ} \mathrm{C}$ under vacuum for $24 \mathrm{~h}$. A couple of electrodes were then interleaved with a porous insulating sheet (separator) and placed into laminated aluminum shell. The prototypes thus fabricated were filled with the corresponding electrolyte and sealed. As an electrolyte, the solution of $1.3 \mathrm{M} \mathrm{Et}_{3} \mathrm{MeNBF}_{4}$ in acetonitrile was used in UC prototypes, while 1.5 M LiTFSI (produced by Solvay) in acetonitrile was used in hybrid devices. In some cases, an additional reference electrode was embedded in the cell. All the assembly operations were carried out in a Vigor glove box filled with dry argon gas. Larger devices comprising a number of positive and negative electrodes connected in parallel and forming a stack were assembled at Yunasko Pilot Plant according to the same technology.

\subsection{Prototype Performance Measurements}

CV (cyclic voltammetry) and EIS (electrochemical impedance spectroscopy) measurements were carried out with the help of Voltalab-80 PGZ-402 unit. Galvanostatic charge-discharge cycling with the help of Arbin SCT 5-25 and Arbin BT-2000 testing units was also used to measure the capacitance and internal resistance of lab test prototypes and large devices. The $\mathrm{CV}$ measurements in two-electrode cells were mostly carried out within the voltage range of $0-3.3 \mathrm{~V}$ with the scan rate of $10 \mathrm{mV} \cdot \mathrm{s}^{-1}$. Three-electrode $\mathrm{CV}$ measurements were also used in order to study the behavior of some active materials in either positive or negative potential range. All the measurements were carried out between $25^{\circ} \mathrm{C}$ and $100^{\circ} \mathrm{C}$, and special life cycle tests were carried out at $60^{\circ} \mathrm{C}$ [3].

The $R_{\text {in }}$ (internal resistance) values were determined using Arbin $I R$ pulse algorithm (where $I$ is the current value and $R$ is the resistance value) with the pulse duration being of $10 \mathrm{~ms}$. The values of capacitance $(C)$ of UC and capacity $(Q)$ of hybrid devices were calculated from galvanostatic cycling results. 


\section{Results and Discussion}

\subsection{UC Inner Resistance, Power Capability and Safety}

From the very beginning, it should be noted that, in the large UC prototypes with their capacitance exceeding $100 \mathrm{~F}$, which are presented and discussed below, commercially available nanoporous carbons have been used as active electrode materials. Some more "exotic" materials like carbide derived carbons [4], carbon nanotubes or graphene have also been tested, but for comparison purposes only. To effectively reduce the $\mathrm{UC}$ inner resistance we have thoroughly analyzed and reduced all the contributions to the total resistance. In particular, the contact resistance at the active electrode layer-Al current collector interface was reduced to values not exceeding $0.01 \Omega \cdot \mathrm{cm}^{2}$ due to the electric spark treatment of the current collector surface with graphite electrode $[1,2]$. In the course of that treatment, the micron-size graphite particles were spot-fused into the collector and destroyed the oxide layer on the Al foil surface forming a good contact between $\mathrm{Al}$ metal and graphite that turned out to be stable over the entire UC lifetime. Besides, we have recently developed electrochemical and NMR (nuclear magnetic resonance) techniques to measure the electrolyte mobility in carbon nanopores in order to best match the electrode materials with organic electrolytes [5]. Due to such preliminary selection of both positive and negative electrode materials, the UC cell design was optimized so as to reduce the electrolyte in-pore resistance and thus to significantly increase the UC power capability and efficiency.

As a general result, carbon-carbon UC devices of $480 \mathrm{~F}, 750 \mathrm{~F}$ and 1,200 F, which were manufactured according to the developed methodology, demonstrated the inner resistance of $0.3-0.1 \mathrm{~m} \Omega$ with their time-constant ${ }^{1}$ of about $0.15 \mathrm{~s}$. This is by far the lowest value for today, as was confirmed by

\footnotetext{
${ }^{1}$ The capacitor time-constant is the product of resistance $R$ (in $\mathrm{Ohm}$ ) and capacitance $C$ (in Farad), or so-called RC-constant.
}

comparative tests performed by Dr. Burke, et al. [6, 7], and recently by Dr. Miller, et al. [8]. That low inner resistance can provide (and does provide) a very high efficiency of our UC devices - the efficiency typically reaches 97\%-98\% even under high loads [9]. That low resistance and high efficiency can play an important role in UC applications in KERS or in stop \& start systems in micro-hybrids.

\subsection{UC Parallel Combination with Battery}

Another advantage of UC devices with very low inner resistance is related with their effective support of batteries in parallel combinations. An example can be seen in Fig. 1 (Figs. 1a and 1b), wherein, we compare the effectiveness of two different UC modules in unloading the same battery during the model engine start event. In this computer simulation, the UC modules have the same capacitance but different inner resistance and, hence, different RC-constants. Fig. 1a illustrates the behavior of the battery-UC parallel combination with our UC module having the RC-constant of $0.15 \mathrm{~s}$, and Fig. 1b illustrates the same combination if the best competing UC module with the RC-constant of $0.48 \mathrm{~s}$ is used.

As can clearly be seen from this comparison, the Yunasko device (Fig. 1a) provides more effective performance of the entire system, in particular, the higher starting total current of about 400 A flowing through the load and more effective unloading the battery. The latter, if being realized, can prolong the battery life and provide its safe operation.

The robustness and safety of our UC devices was also confirmed by abuse tolerance tests like nail penetration or overvoltage according to the standards [10]. During the numerous nail penetration tests, we have never observed any flame or anything looking like thermal runaway. The temperature increase for 1,200 $\mathrm{F}$ cells did not exceed $45^{\circ} \mathrm{C}$ if the test was started at room temperature. To see the effect of overvoltage, we cycled the cells up to 3.3-3.5 V over tens of hours and also at elevated temperature up to $70{ }^{\circ} \mathrm{C}$. Under 


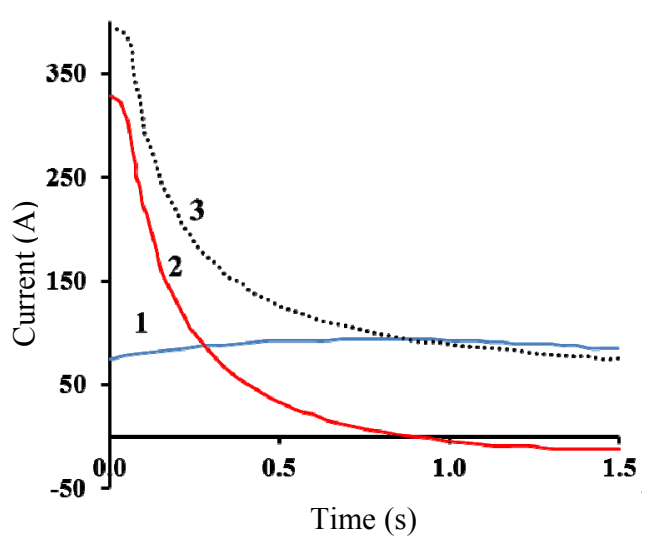

(a)

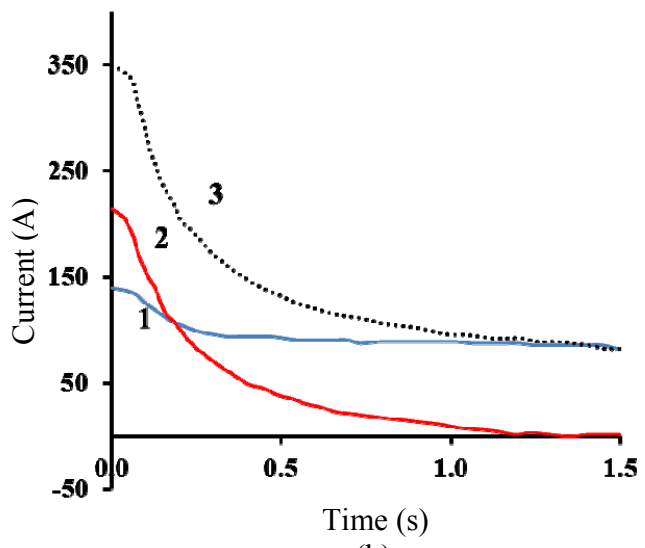

(b)

Fig. 1 Parallel combination of two UC modules of the same size but of different inner resistance with Li-ion battery: (a) UC module: 25 F, $6 \mathrm{~m} \Omega$; (b) UC module: $25 \mathrm{~F}, 19 \mathrm{~m} \Omega$. 1-current flowing through Li-ion; 2-current flowing through UC; 3 -total current.

those severe conditions, we could observe more or less (depending on test duration) deterioration of the UC characteristics but never flame or explosion. Short circuiting the cells or discharging them to zero voltage did not change their performance significantly.

\subsection{An Increase in UC Energy-Hybrid Devices}

Hybrid devices were first developed about 20 years ago by ESMA in Russia [11], and their devices used a combination of a negative electrode made of activated carbon material and a nickel hydroxide positive electrode reacting in aqueous alkaline electrolyte. Thus, the device can be considered as a hybrid of UC and alkaline (or NMH (nickel-metal-hydride)) battery. The technology provides about two times more energy density than conventional carbon-carbon UC devices, though at the expense of lower power density.

A promising approach, which appeared about 10 years ago, includes hybridization of $\mathrm{UC}$ and $\mathrm{Li}$-ion technologies [12]. A group of devices with slightly different design is normally called by the general name of LIC (lithium ion capacitor). In this case, negative electrode and electrolyte are typical for Li-ion technology with Li-ions intercalating into and de-intercalating from the graphite anode. In some recent, LIC technologies LTO is also used as an anode material [13]. Positive electrode is fabricated from activated carbon or other material typical for the UC technology, sometimes with addition of lithiated metal oxides typical for Li-ion technology [13]. The LIC rated voltage reaches $3.8 \mathrm{~V}$ and is higher than that of UC cells and nickel-alkaline hybrids mentioned above, and the general state-of-art of LIC hybrid devices can be characterized by the energy density of about 10-15 Wh/kg.

Our approach to further increase the energy density of hybrid devices, while keeping at the same time high power capability and high efficiency, can be described as follows [14]:

- Both positive and negative electrodes contain the components of Li-ion and UC technology, e.g., a negative electrode is a mixture of LTO and activated carbon powders, and a positive electrode is a mixture of various lithiated metal oxides and/or phosphates and activated carbon powders. Of course, some conductive additives like carbon black or graphite and a binder can also be added to the mixture. NOTE: this design can be defined [15] as a "parallel hybrid" in contrast to series connection of UC and Li-ion electrodes in typical LIC technologies;

- Mass ratio of various electrode components should be chosen properly in order to balance the charge-discharge characteristics and to provide smooth charge-discharge curves of positive and negative electrodes; 
- Various organic electrolytes can be used, but LiTFSI (lithium bis(trifluoromethane) sulfonimide) in acetonitrile is preferred to keep the high conductivity and in-pore mobility resulting in low inner resistance of a hybrid device.

To compare our UC and hybrid technologies, Fig. 2 illustrates some typical discharge curves of UC and hybrid prototypes of the same mass of $83 \mathrm{~g}$. With this mass, the UC prototype had the capacitance of $450 \mathrm{~F}$, and the hybrid one had the capacity of $1.3 \mathrm{Ah}$. As can be seen from Fig. 2, hybrid device can provide $8+$ longer discharge time than a UC one even at high rates (NOTE: 100 A corresponds to $77 \mathrm{C}$ ). Today, these hybrid devices can provide the energy density up to $37 \mathrm{Wh} / \mathrm{kg}$ keeping at the same time the high power capability (about $4 \mathrm{~kW} / \mathrm{kg}$ at $95 \%$ efficiency) typical for the most of UC devices available in the market. Besides, the discharge curve has a plateau similar to that in battery technologies.

On the other hand, our observations show that, the gain in energy density is normally accompanied by the cycle life reduction - see Fig. 3, wherein, the typical number of deep charge-discharge cycles is plotted in logarithmic scale vs. the energy density for various related technologies, namely, Li-ion battery, hybrid device (Yun-H), LIC device as presented by JM Energy Corporation (www.jmenergy.co.jp) and UC.

A theoretical background of this correlation is beyond the scope of this paper, and just a brief

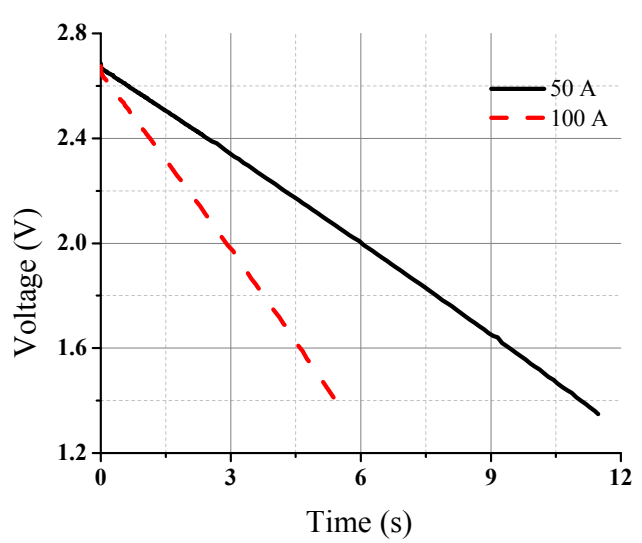

(a) comment is as follows. When changing the technology from left to right (from UC to $\mathrm{Li}$-ion), we increase the role of volume intercalation process in the energy storage. This process provides more energy than the surface charge accumulation in the UC technology, but sooner or later results in distortions in the electrode crystal structure and shortens the battery life cycle.

Though being rather rough, the correlation in Fig. 3 shows that, the energy and power densities and the cycle life of the power supply unit can be varied to best match the performance to the application requirements.

\subsection{Extending the UC Voltage and Temperature Range.}

Today, most of the attempts to increase the UC upper temperature limit and to extend its voltage range are related with the use of ionic liquids. However, bearing in mind the high cost and limited availability of ionic liquids, we are aimed at looking for a reasonably low cost and commercially available organic electrolytes capable of providing the high electrochemical stability and high working temperature of UC devices (at least, up to $3 \mathrm{~V}$ and $100^{\circ} \mathrm{C}$ ). From a variety of aprotic solvents with high boiling points and good electrochemical stability, we have finally chosen sulfolane.

Fig. 4 illustrates typical $\mathrm{CV}$ curves obtained at

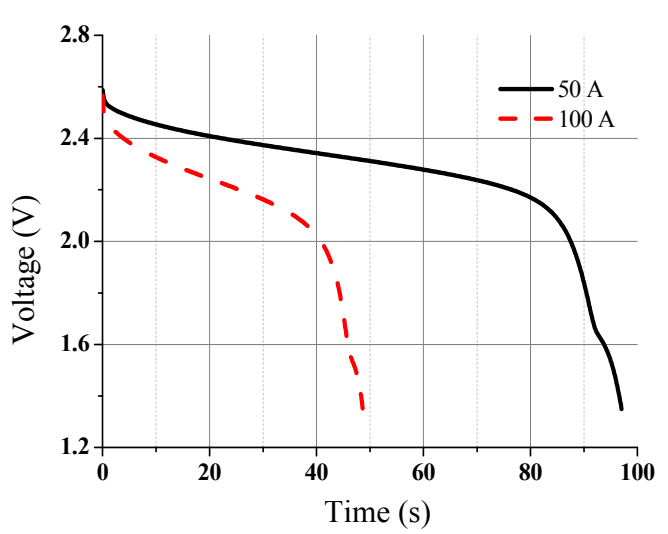

(b)

Fig. 2 Comparison of (a) UC and (b) hybrid device performance: discharge with $50 \mathrm{~A}$ or $100 \mathrm{~A}$, both devices have the same mass of $83 \mathrm{~g}$. 


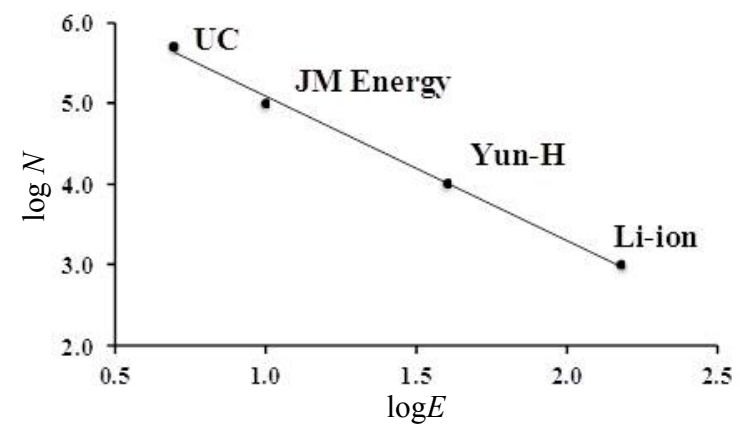

Fig. 3 Plot of typical values (in logarithmic scale) of cycle number vs. the energy density of various related technologies: Li-ion battery (Li-ion), hybrid (Yun-H), LIC (JM Energy), and ultracapacitor (UC).

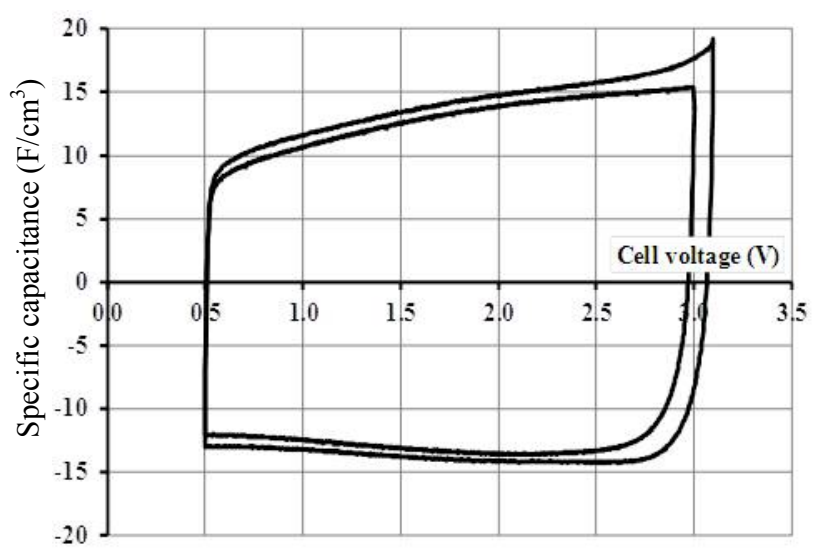

Fig. 4 Cyclic voltammetry curves of a sulfolane based UC prototype at $100{ }^{\circ} \mathrm{C}$, scan rate: $10 \mathrm{mV} \cdot \mathrm{s}^{-1}$.

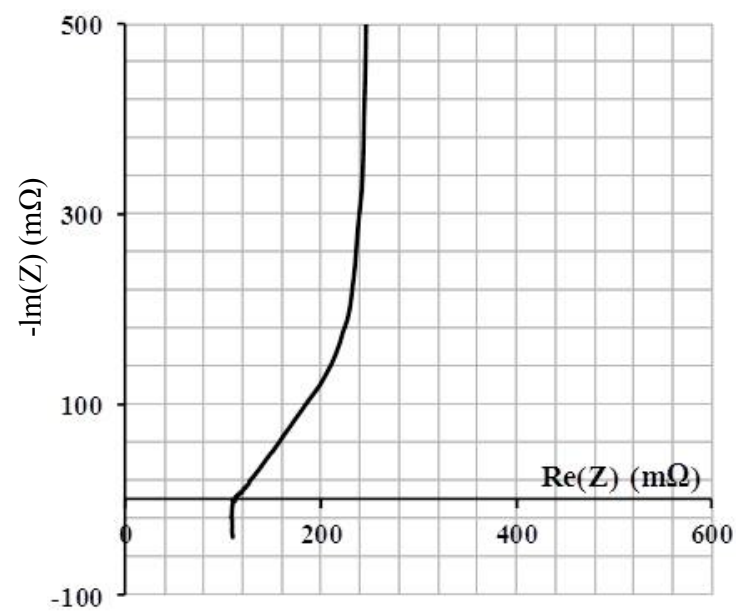

Fig. 5 Nyquist plot of a sulfolane based UC prototype at $100{ }^{\circ} \mathrm{C}$.

$100{ }^{\circ} \mathrm{C}$ for a UC prototype comprising the electrodes based on HDLC 20B STUW active carbon and sulfolane-based electrolyte. As can be seen from Fig. 4, a close to rectangular shape CV curve and sustainable double layer behavior can be maintained up to the voltage value of $3 \mathrm{~V}$. Only above this voltage a small increase in current can be observed and referred to parasitic faradaic processes.

Impedance spectra observed for UC prototypes with sulfolane-based electrolyte at $100{ }^{\circ} \mathrm{C}$ also demonstrate a good capacitor-type behavior [16] with practically vertical line at low frequencies, and close to $45^{\circ}$ slope at higher frequencies - see the typical Nyquist plot in Fig. 5.

\section{Conclusions}

- Due to their extremely low inner resistance UC devices can effectively be used in automotive applications, in particular, for load leveling the batteries, in KERS or stop \& start systems. Even lower resistance is desired to increase the efficiency of the systems;

- The UC energy density can substantially be increased due to hybridization of electrochemical system, namely, if both positive and negative electrodes and also electrolyte comprise the active components of Li-ion and UC technologies;

- The use of sulfolane as a solvent enable to extend the UC upper operating temperature up to $100{ }^{\circ} \mathrm{C}$ keeping at the same time high electrochemical stability within $3 \mathrm{~V}$.

\section{References}

[1] Maletin, Y., Shembel, O., Novak, P., Podmogilny, S., Stryzhakova, N., Izotov, V., Mironova, A., and Danilin, V. 2010. Method for fabricating electrodes of low contact resistance for batteries and double layer capacitors. Ukraine Patent 90,448, filed June 25, 2005, and issued May 11, 2010.

[2] Maletin, Y., Podmogilny, S., Stryzhakova, N., Izotov, V., Mironova, A., Danilin, V., and Maletin. A. 2008. Electrochemical double layer capacitor. US Patent 20,080,151,472, filed December 19, 2007, and issued June 26, 2008.

[3] IEC (International Electrotechnical Commission) 62391-2. 2006. Fixed Electric Double-Layer Capacitors for Use in Electronic Equipment, Part 2: Sectional specification-Electric Double-Layer Capacitors for 
Power Application. Geneva: IEC.

[4] Dash, R., Chmiola, J., Yushin, G., Gogotsi, Y., Laudisio, G., Singer, J., Fischer, J., and Kucheyev, S. 2006. "Titanium Carbide Derived Nanoporous Carbon for Energy-Related Applications." Carbon 44 (12): 2489-97.

[5] Maletin, Y., Strelko, V., Stryzhakova, N., Zelinsky, S., Rozhenko, A. B., Gromadsky, D., Volkov, V., Tychina, S., Gozhenko, O., and Drobny. D. 2013. "Carbon based Electrochemical Double Layer Capacitors of Low Internal Resistance.” Energ. Environ. Res. 3 (2): 156-65.

[6] Burke, A. F. 2000. "Ultracapacitors: why, how, and Where is the Technology." J. Power Sources 91 (1): 37-50.

[7] Burke, A., Liu, Z., and Zhao, H. 2014. "Present and Future Applications of Supercapacitors in Electric and Hybrid Vehicles." Presented at the IEEE International Electric Vehicle Conference, Florence, Italy.

[8] Miller, J. R., Butler, S. M., and McNeal, S. 2014. "Life Performance of Large Electrochemical Capacitors." In Proceedings of the 46th Power Sources Conference, 380-1.

[9] Ghosh, S., and Corrigan, D. 2014. Directed Research Report on Performance Comparison of Commercial Supercapacitors. Detroit: Wayne State University.

[10] Idaho National Engineering and Environmental
Laboratory. 2004. FreedomCAR Ultracapacitor Test Manual. Idaho National Engineering Laboratory report.

[11] Stepanov, A. B., Varakin, I. N., Menukhov, V. V., and Klementov, A. D. 2001. Double layer capacitor. US Patent 6,181,546, filed January 19, 1999, and issued January 30, 2001.

[12] Tasaki, S., Ando, N., Nagai, M., Shirakami, A., Matsui, K., and Hato, Y. 2010. Lithium ion capacitor. US Patent 7,697,264, filed October 19, 2005, and issued April 13, 2010.

[13] Du Pasquier, A., Plitz, I., Menocal, S., and Amatucci, G. 2003. "A Comparative Study of Li-ion Battery, Supercapacitor and Nonaqueous Asymmetric Hybrid Devices for Automotive Applications.” J. Power Sources 115 (1): 171-8.

[14] Chernukhin, S., Tretyakov, D., and Maletin, Y. 2014. Hybrid electrochemical energy storage device. US Patent 2014/0,085,773, filed September 25, 2013, and issued March 27, 2014.

[15] Cericola, D., and Kötz, R. 2012. "Hybridization of Rechargeable Batteries and Electrochemical Capacitors: Principles and Limits." Electrochimica Acta 72 (June): 1-17.

[16] Conway, B. E. 1999. Electrochemical Supercapacitors: Scientific Fundamentals and Technological Applications. New York: Kluwer-Plenum Press. 